\title{
Axion-like-particle decay in strong electromagnetic backgrounds
}

\author{
B. King, ${ }^{a, 1}$ B.M. Dillon, ${ }^{b}$ K.A. Beyer ${ }^{c}$ and G. Gregori ${ }^{c}$ \\ ${ }^{a}$ Centre for Mathematical Sciences, University of Plymouth, \\ Plymouth, PL4 8AA, U.K. \\ ${ }^{b}$ Jožef Stefan Institute, \\ Jamova 39, 1000 Ljubljana, Slovenia \\ ${ }^{c}$ Department of Physics, University of Oxford, \\ Parks Road, Oxford, OX1 3PU, U.K. \\ E-mail: b.king@plymouth.ac.uk, barry.dillon@ijs.si, \\ konstantin.beyer@merton.ox.ac.uk, gianluca.gregori@physics.ox.ac.uk
}

ABstract: The decay of a massive pseudoscalar, scalar and U(1) boson into an electronpositron pair in the presence of strong electromagnetic backgrounds is calculated. Of particular interest is the constant-crossed-field limit, relevant for experiments that aim to measure high-energy axion-like-particle conversion into electron-positron pairs in a magnetic field. The total probability depends on the quantum nonlinearity parameter - a product of field and lightfront momentum invariants. Depending on the seed particle mass, different decay regimes are identified. In the below-threshold case, we find the probability depends on a non-perturbative tunneling exponent depending on the quantum parameter and the particle mass. In the above-threshold case, we find that when the quantum parameter is varied linearly, the probability oscillates nonlinearly around the spontaneous decay probability. A strong-field limit is identified in which the threshold is found to disappear. In modelling the fall-off of a quasi-constant-crossed magnetic field, we calculate probabilities beyond the constant limit and investigate when the decay probability can be regarded as locally constant.

Keywords: Beyond Standard Model, Precision QED

ArXiv EPrint: 1905.05201

\footnotetext{
${ }^{1}$ Corresponding author.
} 


\section{Contents}

1 Introduction $\quad 1$

2 Derivation of pseudoscalar decay probability in a plane-wave pulse 4

3 Constant fields $\quad 8$

$\begin{array}{ll}3.1 \text { Below threshold decay } & 9\end{array}$

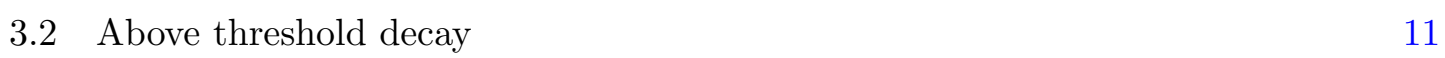

$\begin{array}{ll}3.3 \text { Strong fields, } \chi_{k} \gg 1 & 12\end{array}$

$4 \quad$ Edge effects of static constant fields 13

$\begin{array}{llr}5 & \text { Example experimental scenarios } & 16\end{array}$

$\begin{array}{llr}6 & \text { Summary } & 18\end{array}$

\section{Introduction}

"Naturalness" seems incompatible with the Standard Model (SM) when one considers the "strong-CP" problem, which asks why charge-parity (CP) conjugation invariance is violated so little in the strong sector even though the CP-violating term in the QCD Lagrangian would induce a large but unobserved neutron electric dipole moment. An attractive solution is the Peccei-Quinn (PQ) mechanism which promotes the CP-violating term to be a dynamical parameter that can relax to zero and predicts the existence of a pseudoscalar Nambu-Goldstone boson called the axion [1], which has a weak coupling to photons and electrons, as well as other SM particles. Other beyond-the-StandardModel scenarios predict the existence of light bosonic states that couple weakly to photons and electrons, which are referred to collectively as Axion-Like-Particles (ALPs). They have subsequently been suggested to explain various astrophysical phenomena such as the transparency of the universe to high energy gamma-rays [2-5], and the $3.55 \mathrm{keV}$ galaxy cluster emission line [6-8].

A promising route to detecting ALPs is through their coupling to SM particles in the electromagnetic sector. The coupling of ALPs to the electromagnetic field is exploited in Light-Shining-through-the-Wall (LSW) experiments (for a review see [9]) to convert laser photons in a magnetic background into ALPs, which then propagate through a "wall" and into a low-noise detection region with a background magnetic field. ALPs in the presence of this background field are then reconverted into photons, which provide the experimental signal. The current state-of-the-art LSW experiment is the ALPS I experiment [10], however upgrades to this set-up and other more advanced LSW experiments are planned 
for the future [11, 12]. Helioscope experiments, i.e. CAST [13] and the proposed IAXO experiment [14], also use a similar detection set-up, but since the generation stage occurs in complex astrophysical environments such as in the sun, both production via the di-photon coupling in e.g. the Primakoff process, and production via the electron-ALP coupling in e.g. axionic-Compton emission [15], is being measured. This means that the signal in helioscope experiments, unlike LSW, is also sensitive to the coupling of ALPs to electrons, and a bound on this coupling has been derived by the CAST collaboration [16].

In the current paper we study the process of an ALP decaying to an electron-positron pair in a high-intensity electromagnetic field via a direct coupling of the ALP to electrons. The motivation for our work is: i) to consider the decay of ALPs in a plane-wave background; ii) to look at the effect of the pulse-shape on the background on detection of these ALPs; iii) learn something about field-dressed processes in strong-field QED (SFQED), of which there are very few results in the literature. This study adds to the overall discussion on altering particle decay properties using external electromagnetic (EM) fields and could be of interest for future lab-based ALP searches. In addition to the decay of photons in magnetic [17, 18] and plane-wave [19-21] fields, the decay lifetime of a muon has also been investigated, and shown to be only slightly changed in an EM background in [22, 23], (more recent speculations to the contrary were criticised in the literature [24]). Depending on the set-up of background fields, a magnetic field may enhance or suppress particle production. For example, for constant homogeneous parallel electric and magnetic fields in QED [25] and scalar QED [26], a weak magnetic field has been found to slightly enhance the decay of the vacuum into electron-positron pairs (more details can be found in the review [27]), but for parallel Sauter type electric and magnetic fields [28] and for the decay of a neutral scalar to two charged scalars in a thermal bath [29], to suppress particle production. Also, the effect of a constant magnetic field on the neutral and charged pion masses and decay form factors has recently been investigated [30]. When studying neutral pion decay, it was found (first by Ritus $[22,31]$ ) that a CCF can accelerate the decay into a muon but slow down the decay into an electron (see also [32]). Whilst we note some similarities between ours and Ritus' results for pion decay in a plane-wave background, these are rather limited and of a general nature. Moreover, the processes studied by Ritus do not have the same kinematics of the process as the process $\phi \rightarrow e^{+} e^{-}$, for axions $\phi$, which we are studying here. In [33], the decay of $W^{ \pm}$vector bosons in a CCF was investigated, where it was found that the partial width of the decay $W \rightarrow l \bar{\nu}_{l}$ (for lepton $l$ and corresponding antineutrino $\bar{\nu}_{l}$ ), was a nonmonotonic function of the quantum nonlinearity parameter $\chi$ for $\chi<1$. In [34] the decay $Z^{0} \rightarrow l \bar{l}$ were calculated, where it was found that in the limit of large quantum nonlinearity parameter $\chi \geq 10$, a sizeable contribution of the decay width originates from $Z^{0} \rightarrow t \bar{t}$, which is forbidden in vacuum. Our work complements these studies, as we see that axion decay to a pair is most closely related kinematically to the previous $Z_{0}$ and pion decay studies. We will derive the process in a plane wave of arbitrary pulse form, hence extending the treatment of the background beyond the CCF of previous works. We will use this arbitrary form to model the edge of a magnetic field, and show that decay can occur outside of the field, which is not a result that is obtainable using the locally-constant-field approximation (LCFA). This result will be shown to be due to the 
fact that the contribution from outside the field is vanishing at small interference phase, and therefore the usual Taylor expansion of the LCFA misses this phenomenon. We also study axion decay in a CCF, and find an analogous oscillatory dependence of the decay rate on the quantum nonlinearity parameter for weak fields as well as a monotonic "strongfield" region for large quantum parameter, just as was found in [34] for $Z_{0}$ decay. ([34] also studies the angular dependence of the decay process, which is beyond our current analysis for axions.) One new feature introduced by studying axion decay, is that the mass is a free parameter. Therefore, in a CCF, we were able to identify an exponentially-suppressed tunneling region for axions lighter than the pair rest mass and the transition to a oscillatory dependency on the axion mass for axions heavier than the pair rest mass.

The production of ALPs via their coupling to photons in a circularly-polarised laser beam has been studied in [35]. The production of ALPs in the interactions between electrons and high-intensity electromagnetic fields has been studied previously in [36-39], with ALP-seeded electron-positron pair production in a monochromatic laser background also being considered in [37]. These papers demonstrated how lab-based experiments using high-intensity lasers and electrons may provide lab-based bounds on the ALP electron coupling, complementary to those derived from helioscope experiments. The current paper extends this work by considering the decay of ALPs to electron-positron pairs in quasiconstant magnetic fields, derived as a limit of the case in which the process occurs in a plane-wave electromagnetic background.

This process is relevant for both terrestrial experiments utilising strong magnetic fields for ALP conversion and searches for extraterrestrial ALPs from strongly-magnetised objects $[40]$.

To perform calculations in strong electromagnetic backgrounds, we employ the Furry picture [41]. Solutions to the Dirac equation in a plane-wave electromagnetic background, so-called "Volkov" states, represent the fermions "dressed" in the external electromagnetic field [42]. As such, the derivation of ALP decay rates in quasi-constant electromagnetic fields has much in common with high-intensity QED (reviews can be found in [31, 43-46]), with the decay of photons in a laser background being measured experimentally in the E144 experiment [47, 48]. Due to the immense number of laser photon "probe" particles, most interest in extensions of high-intensity QED has been in the ALP-diphoton coupling. This can manifest itself in the polarisation properties of a photon probe [49-51] or through parametric excitation [52]. Recent calculations have begun exploring the possibility of using the collision of electron bunches with laser pulses to measure the ALP-electron coupling, for example using weak [38], strong [38] and intermediate many-cycle [37] laser pulses, or leveraging collective effects such as coherent emission [39].

The paper is structured as follows. In section 2, we present an example derivation of ALP decay in a plane-wave electromagnetic background with finite support (e.g. a laser pulse), focusing on the decay of a massive pseudoscalar to an electron-positron pair. Derivations for a scalar and a vector boson follow a very similar format and final results for these cases are presented. We then perform a local expansion of the probability and obtain what is often referred to as the "locally-constant-field-approximation" (LCFA) [53-56]. In section 3 we analyse the constant crossed field (CCF) result, which is integrated over the 


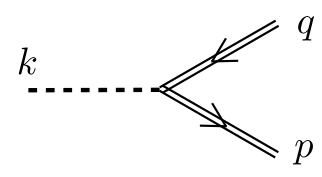

Figure 1. Feynman diagram for pseudoscalar-seeded electron-positron pair production.

non-constant background to form the LCFA. Asymptotic and perturbative limits for belowand above-threshold decay are presented, as is a description of how the non-perturbative pair-creation tunneling and oscillation exponents depend upon the ALP mass, particle energy and field strength. In section 4 we consider the effect of the detector's magnetic field beyond the LCFA and show how large field gradients at the detector edge change the interpretation of a local production of pairs and can influence the total yield. In section 5 we consider two experimental scenarios involving a magnetic field and a laser pulse in the detection region respectively, and take the upcoming LUXE experiment at DESY to provide typical experimental parameters. In section 6 we discuss the results and conclude.

\section{Derivation of pseudoscalar decay probability in a plane-wave pulse}

We begin by considering a pseudoscalar particle, $\phi$, with four-momentum $k$ and mass $m_{\phi}$, decaying to an electron-positron pair in a plane-wave electromagnetic background. The scaled vector potential $a^{\mu}=e A^{\mu}(\varphi)$, where $e$ is the charge of a positron and $A^{\mu}$ the vector potential, depends on a single variable, the phase $\varphi=\varkappa \cdot x$ of the background. The lightlike wavevector of the background $\varkappa$ is transverse to the vector potential, $\varkappa \cdot a=0$, and we represent $a^{\mu}(\varphi)=m \xi(\varphi) \varepsilon^{\mu}$, where $\xi(\varphi)=\xi g(\varphi)$ is the local classical intensity parameter, $g(\varphi)$ is the pulse envelope $(|g(\varphi)| \leq 1), \varepsilon^{\mu}=\left(0, \varepsilon^{\perp}, 0\right)^{\mu}$ is the polarisation and $m$ is the electron mass. We choose a system of co-ordinates in which $\varkappa=\left(\varkappa^{+} / 2\right)(1,0,0,1)$ so that $\varphi=\varkappa^{+} x^{-} / 2=\varkappa^{0} x^{-}$, and use lightfront co-ordinates: $x^{ \pm}=x^{0} \pm x^{3}, x^{\perp}=\left(0, x^{1}, x^{2}, 0\right)$, $x_{ \pm}=x^{\mp} / 2, x_{\perp}=-x^{\perp}$. We will define the seed particle's mass parameter $\delta$, through $\delta^{2}=k^{2} / m^{2}$.

To calculate the probability of decay in the electromagnetic background, we assume the produced fermions are solutions to the Dirac equation in a plane-wave electromagnetic background (Volkov states). This is depicted by the double fermion line in figure 1. The scattering-matrix element for a pseudoscalar is then

$$
\mathrm{S}_{\mathrm{fi}}=i g_{\phi e} \int d^{4} x \phi \bar{\psi}_{p} \gamma_{5} \psi_{q}^{+},
$$

where $g_{\phi e}$ is the electron-pseudoscalar coupling, with Volkov states:

$$
\begin{aligned}
\bar{\psi}_{p} & =\bar{E}_{p}(\varphi) \frac{\bar{u}_{r}(p)}{\sqrt{2 p^{0} V}} e^{i p \cdot x+i S_{p}(\varphi)}, & \psi_{q}^{+} & =E_{-q}(\varphi) \frac{v_{r^{\prime}}(q)}{\sqrt{2 q^{0} V}} e^{i q \cdot x+i S_{-q}(\varphi)}, \\
E_{p}(\varphi) & =1_{4}+\frac{k \phi \phi(\varphi)}{2 \varkappa \cdot p}, & S_{p}(\varphi) & =\int^{\varphi} \frac{2 p \cdot a(\psi)-a^{2}(\psi)}{2 \varkappa \cdot p} d \psi,
\end{aligned}
$$

where we assume that the pseudoscalar is in a plane-wave state, $\phi=e^{-i k \cdot x} / \sqrt{2 k^{0} V}$, has mass $m_{\phi}$, and $V$ is a normalisation volume. 
Integrating eq. (2.1) over the "_" and " $\perp$ " co-ordinates we have

$$
\mathrm{S}_{\mathrm{fi}}=\frac{i g_{\phi e}(2 \pi)^{3}}{\varkappa^{0} \sqrt{8 p^{0} q^{0} k^{0} V^{3}}} \delta^{\perp,-}(p+q-k) \int d \varphi \mathbb{e}^{i \varphi r+i S_{p}(\varphi)+i S_{-q}(\varphi)} \bar{u}_{r}(p) \bar{E}_{p}(\varphi) \gamma^{5} E_{q}(\varphi) v_{r^{\prime}}(q),
$$

where a measure of the lightfront momentum absorbed from the electromagnetic background is given by

$$
r=\frac{p^{+}+q^{+}-k^{+}}{2 \varkappa^{0}} .
$$

To obtain the probability we must square the matrix element

$$
\sum_{\text {spin }} \operatorname{tr}\left|S_{\mathrm{fi}}\right|^{2}=\left[\frac{g_{\phi e}(2 \pi)^{3} \delta^{\perp,-}(p+q-k)}{\varkappa^{0} \sqrt{8 p^{0} q^{0} k^{0} V^{3}}}\right]^{2} \int d \varphi d \varphi^{\prime} \mathrm{Te}^{i r\left(\varphi-\varphi^{\prime}\right)+i\left[S_{p}(\varphi)-S_{p}\left(\varphi^{\prime}\right)\right]+i\left[S_{-q}(\varphi)-S_{-q}\left(\varphi^{\prime}\right)\right]},
$$

where the trace terms are included in the factor

$$
\mathrm{T}=\sum_{r, r^{\prime}, s, s^{\prime}} \operatorname{tr} \bar{u}_{r}(p) \bar{E}_{p}(\varphi) \gamma^{5} E_{q}(\varphi) v_{r^{\prime}}(q) \bar{v}_{s^{\prime}}(q) \bar{E}_{q}\left(\varphi^{\prime}\right) \gamma^{5} E_{p}\left(\varphi^{\prime}\right) u_{s}(p)
$$

Since the electromagnetic background is of a finite extent (disappears at the boundaries of integration), it is more useful to consider probabilities than cross-sections (which would be spacetime-dependent as they depend on the background field strength). The decay probability $\mathrm{P}_{-}$(we use $\mathrm{P}_{-}$for pseudoscalar decay and $\mathrm{P}_{+}$for scalar decay to reflect their behaviour under parity transformation) is defined as $\mathrm{P}_{-}=V^{2} \int \sum_{\text {spin }} \operatorname{tr}\left|\mathrm{S}_{\mathrm{fi}}\right|^{2} d^{3} p d^{3} q /(2 \pi)^{6}$, leading to

$$
\begin{aligned}
\mathrm{P}_{-}= & \frac{g_{\phi e}^{2} \delta^{\perp,-}(0)}{8\left(\varkappa^{0}\right)^{2} k^{0} V} \int \frac{d^{2} p^{\perp} d p^{-}}{q^{-} p^{-}} \theta\left(p^{-}\right) \theta\left(q^{-}\right) \\
& \int d \sigma d \theta \mathrm{T} \exp \left[i \int_{\sigma-\theta / 2}^{\sigma+\theta / 2}\left[\frac{p \cdot a(\phi)}{\varkappa \cdot p}-\frac{q \cdot a(\phi)}{\varkappa \cdot q}-\frac{a^{2}(\phi)}{2} \frac{\varkappa \cdot k}{\varkappa \cdot p \varkappa \cdot q}\right] d \phi+i r \theta\right],
\end{aligned}
$$

where momentum conservation is enforced via $q^{\perp,-}=k^{\perp,-}-p^{\perp,-}$ together with the onshell condition $q^{2}=m^{2}$. In preparation for eventually performing a local expansion, we have defined the average and difference phase variables

$$
\sigma=\frac{\varphi+\varphi^{\prime}}{2} ; \quad \theta=\varphi-\varphi^{\prime}
$$

The probability can be written in a much neater way by observing that

$$
r=\frac{k \cdot p}{\varkappa \cdot q}-\frac{m_{\phi}^{2}}{2 \varkappa \cdot q} \text {. }
$$

We also note

$$
\delta^{\perp,-}(0)=\lim _{l \rightarrow 0} \frac{\delta^{\perp,-}(l) \delta^{+}(l)}{\delta^{+}(l)}=\frac{1}{(2 \pi)^{3}} \frac{V \int d t}{\int d x^{-}}=\frac{V k^{0}}{(2 \pi)^{3} k^{-}}
$$


(where we note that $d \varphi / d \tau$ is a constant in a plane wave, where $\tau$ is the proper time, allowing us to cancel the integrals and introduce corresponding momentum factors).

Then we have

$$
\mathrm{P}_{-}=\frac{g_{\phi e}^{2}}{4\left(\varkappa^{0}\right)^{2} k^{0}(2 \pi)^{3}} \int \frac{d^{2} p^{\perp} d p^{-}}{q^{-} p^{-}} \theta\left(p^{-}\right) \theta\left(q^{-}\right) \int d \sigma d \theta \mathrm{T} \mathrm{e}^{i \theta\left[\left\langle\frac{k \cdot \Pi}{\varkappa \cdot q}\right\rangle-\frac{m_{\phi}^{2}}{2 \varkappa \cdot q}\right]}
$$

where the classical plane-wave momentum of the electron is

$$
\Pi=p-a+\varkappa \frac{2 a \cdot p-a^{2}}{2 \varkappa \cdot p}
$$

and we define the phase-window-average

$$
\langle f\rangle:=\frac{1}{\theta} \int_{\sigma-\theta / 2}^{\sigma+\theta / 2} f(\phi) d \phi .
$$

Performing the spin-sum and the trace we find

$$
\frac{\mathrm{T}}{4}=m^{2}+p \cdot q+\frac{\left[a(\varphi)+a\left(\varphi^{\prime}\right)\right] \cdot p}{2} \frac{\varkappa \cdot k}{\varkappa \cdot p}-\frac{\left[a(\varphi)+a\left(\varphi^{\prime}\right)\right] \cdot q}{2} \frac{\varkappa \cdot k}{\varkappa \cdot q}-\frac{a(\varphi) \cdot a\left(\varphi^{\prime}\right)}{2} \frac{(\varkappa \cdot k)^{2}}{\varkappa \cdot p \varkappa \cdot q} .
$$

To proceed, we wish to perform the $p^{\perp}$ integrals. We note from eq. (2.5) that the exponent is of the form of a Gaussian oscillation in these variables, but the pre-exponent in eq. (2) also contains terms quadratic in $p^{\perp}$ (in $p \cdot q$ ). This would seem to lead to a divergence, however, we will show that the divergent contribution can be reinterpreted as an integral over surface terms, which must disappear.

First of all, we can remove explicit dependence on $q$ (which only remains in the $p \cdot q$ term) by using the trick:

$$
k+\lambda \varkappa=p+q, \quad \lambda=\frac{2 k \cdot p-m_{\phi}^{2}}{2 \varkappa \cdot q},
$$

where $\lambda$ was found from the first equation using the on-shell condition $q^{2}=m^{2}$. Then making the replacement

$$
m^{2}+p \cdot q=k \cdot p \frac{\varkappa \cdot k}{\varkappa \cdot q}-m_{\phi}^{2} \frac{\varkappa \cdot p}{2 \varkappa \cdot q},
$$

the pre-exponent starts to look like the exponent. Writing this explicitly as

$$
\exp [i(\ldots)]=\exp \left\{i\left[\theta\left(\frac{k \cdot p}{\varkappa \cdot q}-\frac{m_{\phi}^{2}}{2 \varkappa \cdot q}\right)+\int_{\sigma-\frac{\theta}{2}}^{\sigma+\frac{\theta}{2}}-\frac{k \cdot a(\phi)}{\varkappa \cdot q}+\frac{\varkappa \cdot k}{2 \varkappa \cdot p}\left(2 a(\phi) \cdot p-a^{2}(\phi)\right) d \phi\right]\right\},
$$

we can make the following replacement in the pre-exponent

$$
\begin{aligned}
\varkappa \cdot k \frac{k \cdot p}{\varkappa \cdot q} \rightarrow & -i \varkappa \cdot k \partial_{\theta}+\frac{\varkappa \cdot k}{2 \varkappa \cdot q} m_{\phi}^{2}+\frac{\varkappa \cdot k}{\varkappa \cdot q} \frac{k \cdot\left(a(\phi)+a\left(\phi^{\prime}\right)\right)}{2} \\
& +\frac{(\varkappa \cdot k)^{2}}{2 \varkappa \cdot q \varkappa \cdot p}\left[-p \cdot\left(a(\phi)+a\left(\phi^{\prime}\right)\right)+\frac{a^{2}(\phi)+a^{2}\left(\phi^{\prime}\right)}{2}\right],
\end{aligned}
$$


which simplifies the pre-exponent considerably such that, in the end, we just have

$$
\frac{\mathrm{T}}{4} e^{i(\ldots)}=\left[\frac{m_{\phi}^{2}}{2}+\frac{(\varkappa \cdot k)^{2}}{2 \varkappa \cdot q \varkappa \cdot p} \frac{\left[a(\phi)-a\left(\phi^{\prime}\right)\right]^{2}}{2}-i \varkappa \cdot k \partial_{\theta}\right] \mathbb{e}^{i(\ldots)} .
$$

If we assume that there can be no contribution to the probability in the infinite past or infinite future, we can discard the derivative term in the pre-exponent and perform the $p^{\perp}$ integrals without encountering a divergence.

Let us write probabilities in the following way:

$$
\mathrm{P}=\frac{g^{2}}{4 \pi} \frac{1}{\eta_{k}} \mathcal{I}
$$

where the coupling and flux prefactors have been separated from the process-dependent integration, $\mathcal{I}$. Then we define the probability $\mathrm{P}_{\text {- }}$ for the decay of a pseudoscalar into an electron-positron pair as $\mathrm{P}_{-}=\left(g^{2} / 4 \pi \eta_{k}\right) \mathcal{I}_{\text {- }}$. Selecting a linearly-polarised background, we then eventually arrive at

$$
\mathcal{I}_{-}=\frac{i}{4 \pi} \int d \sigma d t \frac{d \theta}{\theta+i \varepsilon}\left\{\delta^{2}+\frac{\left[a(\phi)-a\left(\phi^{\prime}\right)\right]^{2}}{2 t(1-t)}\right\} e^{\frac{i \theta \mu(\theta)}{2 \eta_{k} t(1-t)}-\frac{i \theta \delta^{2}}{2 \eta_{k}}}
$$

where we define the Kibble mass factor

$$
\mu(\theta)=1+\left\langle\frac{a}{m}\right\rangle^{2}-\left\langle\left(\frac{a}{m}\right)^{2}\right\rangle
$$

lightfront momentum fraction $t=p^{-} / k^{-}$, and the energy parameter $\eta_{k}=\varkappa \cdot k / m^{2}$. (The energy parameter can be thought of as the squared ratio of the centre-of-mass energy to the pair rest energy, for the case when the seed photon collides with a single background photon to produce a pair.) It is possible to perform the $t$-integral analytically (see e.g. [57])

$\mathcal{I}_{-}=\frac{1}{8 \pi} \int d \sigma \frac{d \theta}{\theta+i \varepsilon}\left\{h(\theta) \delta^{2} \mathrm{~K}_{1}[i h(\theta)]+\left[h(\theta) \delta^{2}+i\left[a(\phi)-a\left(\phi^{\prime}\right)\right]^{2}\right] \mathrm{K}_{0}[i h(\theta)]\right\} e^{-i h(\theta)-\frac{i \theta \delta^{2}}{2 \eta_{k}}}$

where $h(\theta)=-\theta \mu(\theta) / 2 \eta_{k}$ and $\mathrm{K}_{n}(x)$ is the modified Bessel function of second kind [58]. However eq. (2.13) will prove to be the more useful form of the probability for numerical evaluation.

Without further derivation, in the spirit of eq. (2.12), we state that the probability $\mathrm{P}_{+}$ for the decay of a scalar into an electron-positron pair is proportional to the integral:

$$
\mathcal{I}_{+}=\frac{i}{4 \pi} \int d \sigma d t \frac{d \theta}{\theta+i \varepsilon}\left\{4-\delta^{2}+\frac{\left[a(\phi)-a\left(\phi^{\prime}\right)\right]^{2}}{2 t(1-t)}\right\} e^{\frac{i \theta \mu(\theta)}{2 \eta_{k} t(1-t)}-\frac{i \theta \delta^{2}}{2 \eta_{k}}}
$$

and the probability $\mathrm{P}_{\gamma}$ for the decay of an unpolarised massive $\mathrm{U}(1)$ boson is proportional to the integral:

$\mathcal{I}_{\gamma}=\frac{i}{4 \pi} \int d \sigma d t \frac{d \theta}{\theta+i \varepsilon}\left\{2\left(1-\frac{\delta^{2}}{2(1-t)}\right)-\left[a(\varphi)-a\left(\varphi^{\prime}\right)\right]^{2}\left(1-\frac{1}{2 t(1-t)}\right)\right\} e^{\frac{i \theta \mu(\theta)}{2 \eta_{k} t(1-t)}-\frac{i \theta \delta^{2}}{2 \eta_{k}}}$. 


\section{Constant fields}

The Locally Constant Field Approximation (LCFA) allows one to calculate probabilities for processes in non-trivial plane-wave electromagnetic backgrounds by performing a local field expansion of the background, and integrating the resulting constant field result over the non-trivial form of the plane-wave. It has been shown to be a good approximation [53, 59] when the intensity parameter of the background $\xi$, satisfies $\xi \gg 1$, where $\xi^{2}=\langle p \cdot T(\varphi)$. $p\rangle_{\varphi} / m^{2}(\varkappa \cdot p)^{2}$, for massive seed particle four-momentum $p$, stress-energy tensor $T^{\mu \nu}=$ $\left(F^{2}\right)^{\mu \nu}-\eta^{\mu \nu} \operatorname{tr} F^{2} / 4, F$ is the Faraday tensor and $\langle\cdot\rangle_{\varphi}$ implies a cycle-average over the phase $\varphi[60]$. (However, recent analyses of nonlinear Compton scattering hint that the infra-red behaviour is badly approximated by the LCFA $[55,61]$.) (The LCFA is sometimes explained by reference to when a massive seed particle is highly relativistic, the electromagnetic field in the particle's rest-frame is approximately that of a constant-crossed field [31].)

One can acquire the LCFA result from the probability for a process in a plane-wave pulse, such as eq. (2.13), by expanding the exponent in $\theta$ up to $\mathcal{O}\left(\theta^{3}\right)$ which corresponds to the highest power contributing to the constant field case. This amounts to making the replacements

$$
\theta \mu \rightarrow \theta+\frac{f^{2}(\sigma)}{12} \theta^{3} \quad\left(\mathbf{a}(\phi)-\mathbf{a}\left(\phi^{\prime}\right)\right)^{2} \rightarrow-\theta^{2} f^{2}(\sigma),
$$

where $f(\sigma)=\xi^{\prime}(\sigma)$ and the linearly-polarised background can be written $a=-\varepsilon(a \cdot \varepsilon)$ and $-\varepsilon \cdot a^{\prime}(\sigma)=m f(\sigma)$. Using the results:

$$
\begin{aligned}
\int_{-\infty}^{\infty} \frac{d \theta}{\theta+i \varepsilon} e^{i\left(r \theta+c_{3} \theta^{3}\right)} & =-2 \pi i \mathrm{Ai}_{1}\left[\frac{r}{\left(3 c_{3}\right)^{1 / 3}}\right] \\
\int_{-\infty}^{\infty} d \theta \theta \mathbb{e}^{i\left(r \theta+c_{3} \theta^{3}\right)} & =-\frac{2 \pi i}{\left(3 c_{3}\right)^{2 / 3}} \mathrm{Ai}^{\prime}\left[\frac{r}{\left(3 c_{3}\right)^{1 / 3}}\right],
\end{aligned}
$$

for $c_{3} \in \mathbb{R}$, we then find

$$
\mathcal{I}_{-}^{\mathrm{LCFA}}=\int d \sigma d t\left\{\frac{\delta^{2}}{2} \mathrm{Ai}_{1}(z)-\chi_{k}(\sigma) \sqrt{z_{0}} \operatorname{Ai}^{\prime}(z)\right\}
$$

where we define

$$
z_{0}=\left(\frac{\chi_{k}(\sigma)}{\chi_{p}(\sigma) \chi_{q}(\sigma)}\right)^{2 / 3} ; \quad z=z_{0}-\frac{\delta^{2}}{\chi_{k}(\sigma) \sqrt{z_{0}}}
$$

$\chi_{k}(\sigma)=f(\sigma) \eta_{k}$. (Applying the above procedure to the $\mathrm{U}(1)$ case eq. (2.17) and taking the massless limit $\delta^{2} \rightarrow 0$, leads exactly to the QED expression for photon-seeded paircreation [62]. Furthermore the mass-dependent part of the Airy argument has the same form as expected from e.g. the second step of electron-seeded pair-creation, given explicitly in [63].)

Typically, we are interested in lab-based detection of ALPs using constant magnetic fields. Clearly, a constant magnetic field, which can be written $a(\varphi) \sim \varphi$ for spacelike $\left(\varkappa^{2}<0\right)$ wavevector, is relativistically inequivalent to a constant crossed field (CCF), 
which has equal magnitude electric and magnetic fields. However, if we restrict our analysis to highly relativistic seed particles, i.e. with $k^{-} / m \gg 1$, then from their rest frame, a constant magnetic field will appear to be well-approximated by a CCF [64]. This fact, which underlies the Weizsäcker-Williams approximation [65, 66], was recently explicitly shown to hold for nonlinear Thomson scattering in a constant magnetic field [67]. The constant-field limit $a(\varphi) \sim \varphi$ for lightlike wavevector is then the constant crossed field limit.

To approximate the probability in a constant magnetic field using a CCF, we can relate the scaled vector potential, $a$, to the field-strength $F$ by first writing $F$ as

$$
F(\varphi)=\partial_{t} A^{1}(\varphi)=\frac{1}{e} \frac{\partial \varphi}{\partial t} a^{1 \prime}(\varphi)
$$

where we pick the background to be polarised in the 1-direction without loss of generality. To proceed in evaluating $\partial \varphi / \partial t$, we use the same reasoning as in eq. (2), then we see that the non-trivial component of the reduced vector potential, $a^{1}(\varphi)$, can be written

$$
a^{1}(\varphi)=m \xi(\varphi) \rightarrow m \frac{k^{0}}{k^{-}} \frac{L}{\lambda_{\mathrm{C}}} \frac{F_{0}}{F_{\mathrm{Q}}} \varphi
$$

where we have introduced a nominal "frequency" of the constant field $\varkappa^{0}$ to be $\varkappa^{0}=2 \pi / L$ with $L$ being the longitudinal spatial extent of the constant field (formally infinite in the CCF limit), $\lambda_{\mathrm{C}}=2 \pi / \mathrm{m}$ is the Compton wavelength, $F_{0}$ is the amplitude of the field strength, and $F_{\mathrm{Q}}=m^{2} / e$ is the Schwinger limit. Other quantities can then be written independent of any external field frequency:

$$
\chi_{k}(\sigma) \rightarrow \chi_{k}=f \eta_{k}=\frac{F}{F_{\mathrm{Q}}} \frac{k^{-}}{m} ; \quad \frac{1}{\eta_{k}} \int d \sigma \rightarrow \frac{m}{k^{-}} \frac{L}{\lambda} .
$$

Then we see:

$$
\mathrm{P}_{-}^{\mathrm{CCF}}=\frac{g^{2}}{4 \pi} \frac{m}{k^{-}} \frac{L}{\lambda} \mathrm{R}_{-}^{\mathrm{CCF}}\left(\chi_{k}, \delta^{2}\right),
$$

where all the non-trivial dependency on experimental parameters is contained within the function $\mathrm{R}_{-}^{\mathrm{CCF}}$, which is the rate per unit detector length (measured in units of the reduced Compton wavelength).

In relation to the ALP mass and the field strength we can identify three distinct regimes for creation of electron-positron pairs: i) below threshold, $\delta^{2}<4$, where the process is forbidden in the limit of zero field and hence is field-induced; ii) above threshold, $\delta^{2}>4$, where the process is field-assisted and can proceed in the zero-field limit, and iii) strong field $\chi \gg 1$, where decay is so likely, there is no threshold behaviour anymore. Plotting the dependency of $\mathrm{R}_{-}^{\mathrm{CCF}}$ on $\chi_{k}$ in figure 2 for different axion mass parameter, $\delta^{2}$, one can clearly see where these three regions occur.

\subsection{Below threshold decay}

Below-threshold, pair creation can only occur as a tunneling process and hence is exponentially suppressed. This situation is very similar to studies on photon-seeded paircreation [59,68], and so we will not analyse it in great detail. However, we highlight the 

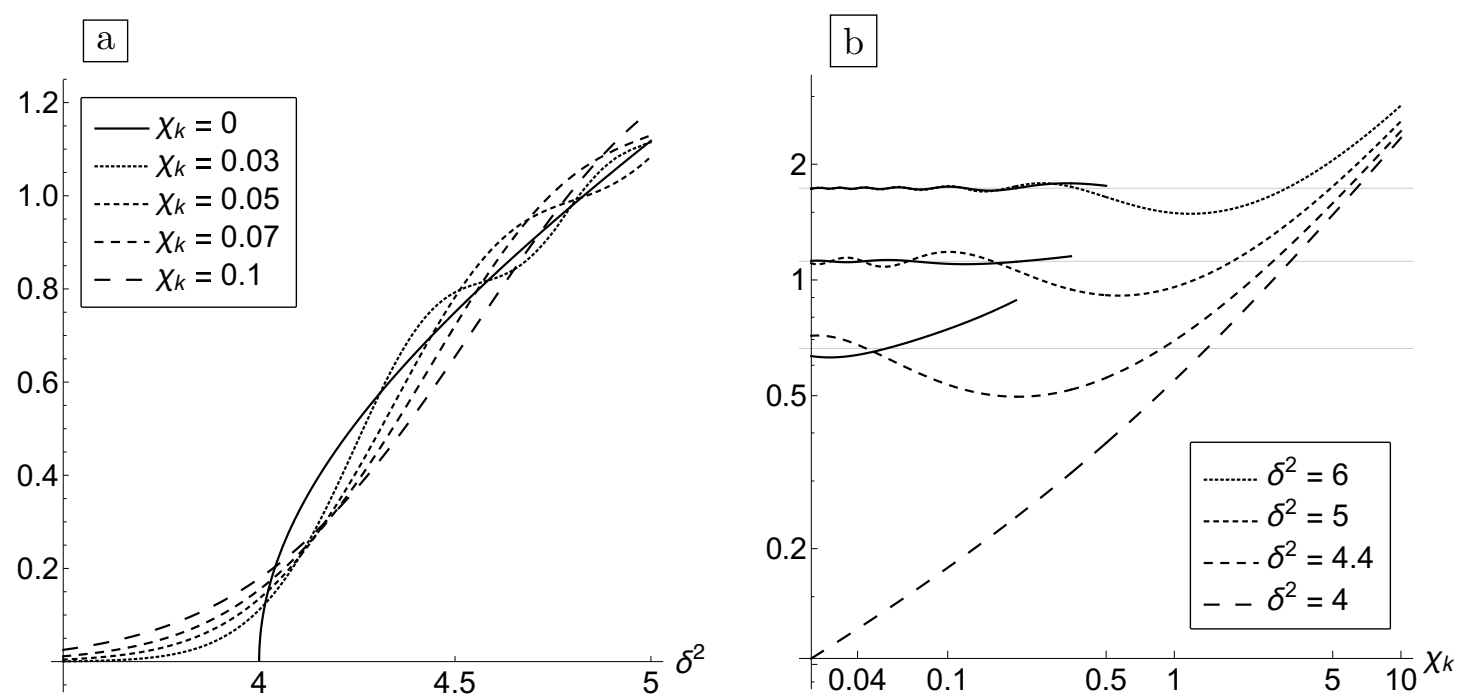

Figure 2. Plots of $\mathrm{R}_{-}^{\mathrm{CCF}}$ for various seed-particle masses. a) the effect of the threshold when $\chi_{k} \ll 1$ - exponential suppression below the threshold and an oscillatory dependence on $\delta^{2}$ above it. b) for small $\chi_{k}$, when the decay is above-threshold, the probability oscillates about the vacuum values (shown as grey horizontal lines) and in the below-threshold regime, the probability is exponentially suppressed. For large $\chi_{k}$, the distinction between above- and below- threshold is lost and the dependence on particle mass disappears. In b), black solid lines are the asymptotic results, which become more accurate for heavier masses and smaller $\chi_{k}$.

difference that a massive seed particle brings, by calculating the asymptotic and perturbative limits of $\mathrm{R}_{-}^{\mathrm{CCF}}$.

In a CCF, the exponent in eq. (2.13) (and eqs. (2.16) and (2.17)), can be written, with a change of variable to make the dependency on $\chi_{k}$ manifest, $\theta \rightarrow 2 \vartheta / \xi$, as:

$$
\exp \left[\frac{i \vartheta}{\chi t(1-t)}\left(1+\frac{\vartheta^{2}}{3}-\delta^{2} t(1-t)\right)\right] .
$$

In the subthreshold case, $\delta^{2}<4$, we note that $1-\delta^{2} t(1-t)>0$. Then in this case, the turning points of the exponent always lie on the imaginary axis. Rotating the integration contour with $\vartheta \rightarrow i \vartheta$, yields a real exponent of the form $\sim \vartheta-c \vartheta^{3}$, allowing us to use the Laplace method. There are two turning points and one is subdominant.

We then find for $\delta^{2}<4$ and $\chi_{k} \ll 1$ :

$$
\mathrm{R}_{-}^{\mathrm{CCF}} \sim \chi_{k} \frac{\sqrt{3}}{4 \sqrt{2}}\left(1+\frac{\delta^{2}}{8}\right)^{-1 / 2}\left(1+\frac{\chi_{k}}{2} \frac{\delta^{2}}{4-\delta^{2}}\right) \mathrm{e}^{-\frac{8}{3 \chi_{k}}\left(1-\frac{\delta^{2}}{4}\right)^{3 / 2}} .
$$

We notice the familiar $-8 / 3 \chi_{k}$ tunneling exponent has been shifted by the seed particle mass, where the tunneling behaviour clearly disappears as the mass approaches the threshold $\delta^{2} \rightarrow 4$. We can understand this in an intuitive way by using arguments based on energy-momentum conservation [69], recently applied to high-intensity laser-based QED [70]. The energy of the produced electron is:

$$
\mathcal{E}_{p}(t)=\sqrt{(\mathbf{p}-e \mathbf{A})^{2}+m^{2}}=\sqrt{p^{2}+m^{2}+e^{2} F^{2} t^{2}},
$$


where in the last equality, we have used the fact that the background field is constant. Assuming all particles involved in the decay are highly relativistic, we can see that the energy change is:

$$
\Delta \mathcal{E}(t)=\mathcal{E}_{p}(t)+\mathcal{E}_{k-p}(t)-\mathcal{E}_{k} \approx \frac{2 m^{2}}{k}\left\{\left[1+\frac{(e F t)^{2}}{m^{2}}\right] \frac{k^{2}}{4 p(k-p)}-\frac{\delta^{2}}{4}\right\} .
$$

We can approximate the form of the rate for the process to occur using the WKB method [71]:

$$
\frac{d \mathrm{P}}{d t} \sim \exp \left[i \int_{0}^{t} \Delta \mathcal{E}\left(t^{\prime}\right) d t^{\prime}\right] \sim \mathrm{R}_{-}^{\mathrm{CCF}}
$$

For a tunneling process, we can approximate this integral by using the saddle-point method, and integrating to $t_{*}$ where $t=t_{*}$ is the shortest time for which $\Delta \mathcal{E}(i t)=0$. The smallest energy difference corresponds to an equal distribution of the initial energy and momentum $p=k / 2$, from which it follows that the tunneling time is:

$$
t_{*}=\frac{m}{e F} \sqrt{1-\frac{\delta^{2}}{4}}
$$

and using eq. (3.11), we indeed find that:

$$
\mathrm{R}_{-}^{\mathrm{CCF}} \sim \exp \left[-\frac{8}{3 \chi_{k}}\left(1-\frac{\delta^{2}}{4}\right)^{3 / 2}\right]
$$

Therefore, we can be somewhat confident that we have the correct tunneling exponent.

\subsection{Above threshold decay}

Above threshold, $\delta^{2}>4$, a region of the $t$-integration exists where $1-\delta^{2} t(1-t)<0$. Then in this case, two turning points of the exponent eq. (3.7) appear, with opposite sign, on the real $\vartheta$ axis.

Then applying the method of stationary-phase in $\vartheta$, we acquire a final integral in $t$, with an oscillating exponent which also has turning points on the real axis. Of those, two turning points conspire to produce a cosine, and the third turning point gives a constant term, which is where the zero-field contribution originates. Altogether we find when $\chi_{k} \ll 1$, $\delta^{2}>4$, that:

$$
\mathrm{R}_{-}^{\mathrm{CCF}} \sim-\frac{\chi_{k} \sqrt{3}}{8}\left(1+\frac{\delta^{2}}{8}\right)^{-1 / 2}\left(4+\frac{\delta^{2}}{\delta^{2}-4}\right) \cos \left[\frac{8}{3 \chi_{k}}\left(-1+\frac{\delta^{2}}{4}\right)^{3 / 2}\right]+\frac{1}{2} \sqrt{\delta^{2}\left(\delta^{2}-4\right)} .
$$

We see that in this low- $\chi_{k}$ limit, the probability for decay oscillates as the field-strength is varied. This is demonstrated for various axion masses in figure $2 \mathrm{a}$, where we plot this transition, and the dependency of $\mathrm{R}_{-}^{\mathrm{CCF}}$ on ALP mass parameter, $\delta^{2}$, where the threshold effect can clearly be seen. 
The zero-field result in eq. (3.12) must, of course, be independent of the form of the background field. By taking the limit $a \rightarrow 0$ in eq. (2.13), we find the same result, which can be written as:

$$
\mathrm{P}_{-}(\xi \rightarrow 0) \rightarrow \frac{g^{2}}{4 \pi} \frac{m T}{2} \sqrt{\delta^{2}\left(\delta^{2}-4\right)}
$$

where $T=\int d t$.

\subsection{Strong fields, $\chi_{k} \gg 1$}

In this parameter region, one can simply perturbatively expand eq. (3.3) in the small parameter $1 / \chi_{k}$ since the Airy argument is given by:

$$
z=\frac{1}{\chi_{k}^{2 / 3}}\left(\frac{1}{t(1-t)}\right)^{2 / 3}\left[1-\delta^{2} t(1-t)\right]
$$

At a given $\chi_{k} \gg 1$, this perturbative expansion decreases in accuracy for increasing $\delta^{2}$. Suppose $\delta^{2} \gg 1$, then $z \sim \delta^{2} / \chi_{k}^{2 / 3}$. So in the perturbative limit, we must also assume that $\chi_{k}^{2 / 3} \gg \delta^{2}$.

After a straightforward integration in $t$, we find:

$$
\mathrm{R}_{-}^{\mathrm{CCF}}\left(\chi_{k}, \delta^{2}\right) \approx \frac{2^{4 / 3} \pi \chi_{k}^{2 / 3}}{3^{1 / 3} \Gamma\left(\frac{1}{6}\right) \Gamma\left(\frac{7}{6}\right)}+O\left(\chi_{k}^{0}\right) ; \quad O\left(\chi_{k}^{0}\right)=\frac{\delta^{2}}{3}+O\left(\chi_{k}^{-2 / 3}\right) .
$$

We have included the next-to-leading-order term in $\chi_{k}^{2 / 3}$ to show that, in the limit $\chi_{k}^{2 / 3} \gg$ $\delta^{2}$, the mass of the seed particle ceases to play a role, and in general, the concept of a threshold disappears as $\chi_{k}$ increases towards $\chi_{k} \gg 1$.

Again, the functional dependence of eq. (3.13) can be understood by using intuitive methods $[69,70]$. In this case, the process is above-threshold and so the rate is simply proportional to $g^{2}$ (because one vertex) and $1 / t_{q}$ (because of dimensions), where $t_{q}$ is the quantum time fulfilling $t_{q}=1 / \Delta \mathcal{E}\left(t_{q}\right)$ from the uncertainty relation. (Since the process is quantum, the classical timescale $t_{c l}$ given by $e F\left(t_{c l}\right) t_{c l}=m$ should not be significant.) Using eq. (3.10) generates a cubic in $t_{q}$, and the one real root leads to the relation:

$$
\mathrm{R}_{-}^{\mathrm{CCF}} \sim \chi_{k}^{2 / 3}+O\left(\chi_{k}^{0}\right) ; \quad O\left(\chi_{k}^{0}\right) \sim\left[1-\frac{\delta^{2}}{4}\right]+O\left(\chi_{k}^{-2 / 3}\right)
$$

Also here, we include the next-to-leading order, to show the dependency on the mass. Although the pseudoscalar mass term in our result eq. (3.13) depends on the mass as $\sim \delta^{2}$ and not $\sim\left(1-\delta^{2} / 4\right)$ as in the intuitive method, it is not entirely surprising that this term differs since it originates from the parity-dependent term in the trace, which takes a different form whether dealing with e.g. a photon, scalar or pseudoscalar and nowhere have we inserted the fact that we are dealing with a pseudoscalar in this intuitive picture. (Indeed from eq. (2.16) the scalar mass term is $\sim-\left(1-\delta^{2} / 4\right)$.) 


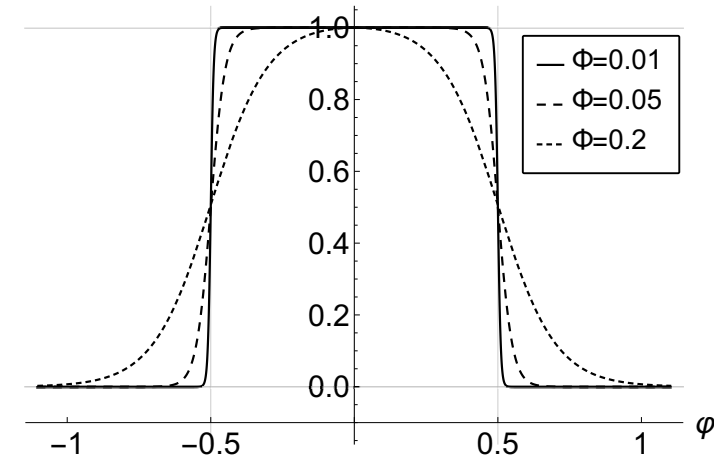

Figure 3. Magnetic part of a constant crossed field, scaled so the maximum is unity. For highlyrelativistic axions, the crossed field produces an equivalent effect to entering a constant homogeneous magnetic field. The limit $\Phi \rightarrow 0$ gives the top-hat function.

\section{Edge effects of static constant fields}

We recall that we are working in the highly-relativistic regime, where processes are wellapproximated by replacing a constant field with a CCF. Therefore, to represent the edge of a quasi-constant magnetic field, we choose a field of plane-wave form:

$$
B(\varphi)=\frac{F_{0}}{1+\tanh \left(-\varphi_{0} / \Phi\right) \tanh \left(\varphi_{1} / \Phi\right)}\left[1+\tanh \left(\frac{\varphi-\varphi_{0}}{\Phi}\right) \tanh \left(\frac{\varphi_{1}-\varphi}{\Phi}\right)\right]
$$

where $\varphi=\varphi_{0,1}$ (and $\varphi_{1}>\varphi_{0}$ ) are the phase positions of the two "edges" of the field and $\Phi$ is a sharpness parameter. We choose, without loss of generality, $\varphi_{0}=-0.5, \varphi_{1}=0.5$, and exhibit the form of $B$ for various sharpness parameters in figure 3 . The potential is then derived from this form of the field numerically by solving $a^{\prime}(\varphi)=m\left(k^{0} / k^{-}\right)\left(L / \lambda_{\mathrm{C}}\right) B(\varphi) / F_{\mathrm{Q}}$, which reduces to eq. (3.5) in the constant-field limit.

To apply the result for the probability for axion decay in a plane-wave field eq. (2.13) to a quasi-static magnetic field in the highly-relativistic regime, we use eq. (3.5), whence it follows $\xi=(L / \lambda)\left(F_{0} / F_{\mathrm{Q}}\right)$. For a magnetic field of $F_{0}=1 \mathrm{~T}$ and length $L=1 \mathrm{~m}$, $\xi \sim O\left(10^{2}\right)$, however the frequency scale $\varkappa^{0} / m=2 \pi \lambda / L \ll 1$, so in this case $\eta_{k} \ll 1$ and hence $\chi_{k}=\xi \eta_{k} \ll 1$. Therefore, following from the results in figure 2, for a terrestrial magnetic field, we expect the pair-decay to only occur for axions with a mass that is close to, or already above threshold. Still, we will begin by analysing the below-the-threshold case as it gives a clearer demonstration of the effect of field gradients introduced by having a rapid drop-off at the end of the magnetic field.

In this case, the numerical evaluation of eq. (2.13) is non-trivial, because the constantfield part is not absolutely convergent. We found it was sufficient to integrate by parts once in $\theta$ and to numerically evaluate the resulting, absolutely convergent integral.

Upon comparison with the LCFA, we expect that when $\xi \Phi \gg 1$, there may be a discrepancy. 

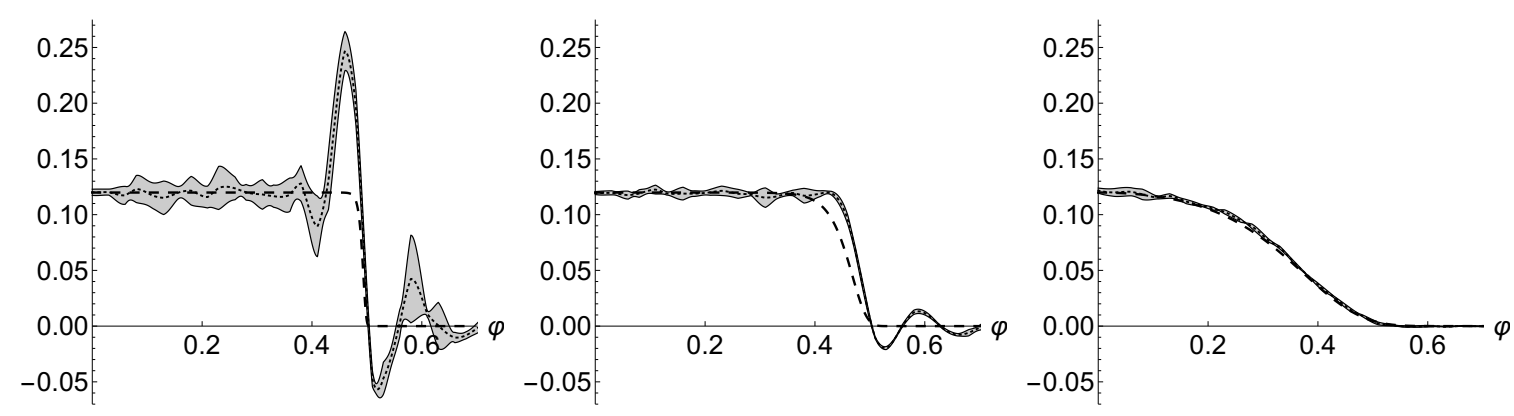

Figure 4. Plots of $\mathcal{I}_{-}$for a field when $\delta^{2}=0, \xi=10, \eta_{k}=0.1$, for three cases: $\Phi=0.2, \Phi=0.05$ and $\Phi=0.01$ respectively from left to right. The dashed line is the LCFA, the dotted line is the mean numerical result and the gray area marks out one standard deviation from the mean. The numerics were run five times for each plot, and each numerical evaluation randomly allocated 1000 points in $\theta$ between 0 and 1 , which were distributed with a cubic weighting towards $\theta=0$.

We can justify the LCFA by applying the substitution $\theta \rightarrow y / \xi$ in eq. (2.13) to give:

$$
\mathcal{I}_{-}=\frac{i}{4 \pi} \int d \sigma d t \frac{d y}{y}\left\{\delta^{2}+\frac{\left[a\left(\sigma+\frac{y}{2 \xi}\right)-a\left(\sigma-\frac{y}{2 \xi}\right)\right]^{2}}{2 t(1-t)}\right\} e^{\frac{i y}{2 \chi_{k}}\left[\frac{\mu\left(\frac{y}{\xi}\right)}{t(1-t)}-\delta^{2}\right]}
$$

Then if $\xi \gg 1$, we expect a Taylor expansion of functions in $y / \xi$ - for example the dimensionless Kibble Mass, $\mu$ - to be the basis of a good approximation. The conditions that powers of $y$ higher than $y^{3}$ can be discarded - and hence the LCFA used - include such inequalities as $\left[(y / \xi)\left(a^{\prime \prime}(\sigma) / a^{\prime}(\sigma)\right)\right]^{2} \ll 1,\left[(y / \xi)^{2}\left(a^{\prime \prime \prime}(\sigma) / a^{\prime}(\sigma)\right) \ll 1\right.$, and for these terms to make a difference, the probability must not be already vanishingly small when these inequalities are violated. It then follows that $(\xi \Phi)^{-2} \ll 1$ for the LCFA to be valid. For these parameters, where we have chosen to associate the external-field frequency $\varkappa^{0}$ with $2 \pi / L$, if the ALP collides head-on with the wavevector of the magnetic field, $\Phi$ then represents some length or duration, $\Delta$, over which the field falls off at its edges. Then LCFA is valid when:

$$
\left(\frac{1}{\xi} \frac{L}{\Delta}\right)^{2} \ll 1 ; \quad\left(\frac{\lambda_{\mathrm{C}}}{\Delta} \frac{F_{\mathrm{Q}}}{F_{0}}\right)^{2} \ll 1,
$$

where $\lambda_{C}$ is the Compton wavelength of an electron. Therefore, the weaker the field, the more important its shape. This is somehow intuitive: a weaker field has a lower intensity and so the approximation that it is locally constant should be worse. For a $1 \mathrm{~T}$ magnet, this corresponds to a field edge of approximately $\Delta^{2} \ll 10^{-4} \mathrm{~m}^{2}$.

We demonstrate the effect of the sharpness of the field in figure 4 for $\xi=10, \eta_{k}=0.1$ (a much higher value than from an ALP in a homogeneous magnetic field in the lab, which would require a $100 \mathrm{TeV}$ ALP in a $1 \mathrm{~T}$ magnet), so that $\chi_{k}=1$ and pair-creation is appreciable.

The numerical curves in figure 4 are also seen to oscillate around zero outside of the magnetic field. It is known [55] that $\partial \mathrm{P} / \partial \sigma$ (we recall the definition of $\sigma$ in eq. (2.6) as the seed particle's average phase position) does not have to be positive, as long as the total 

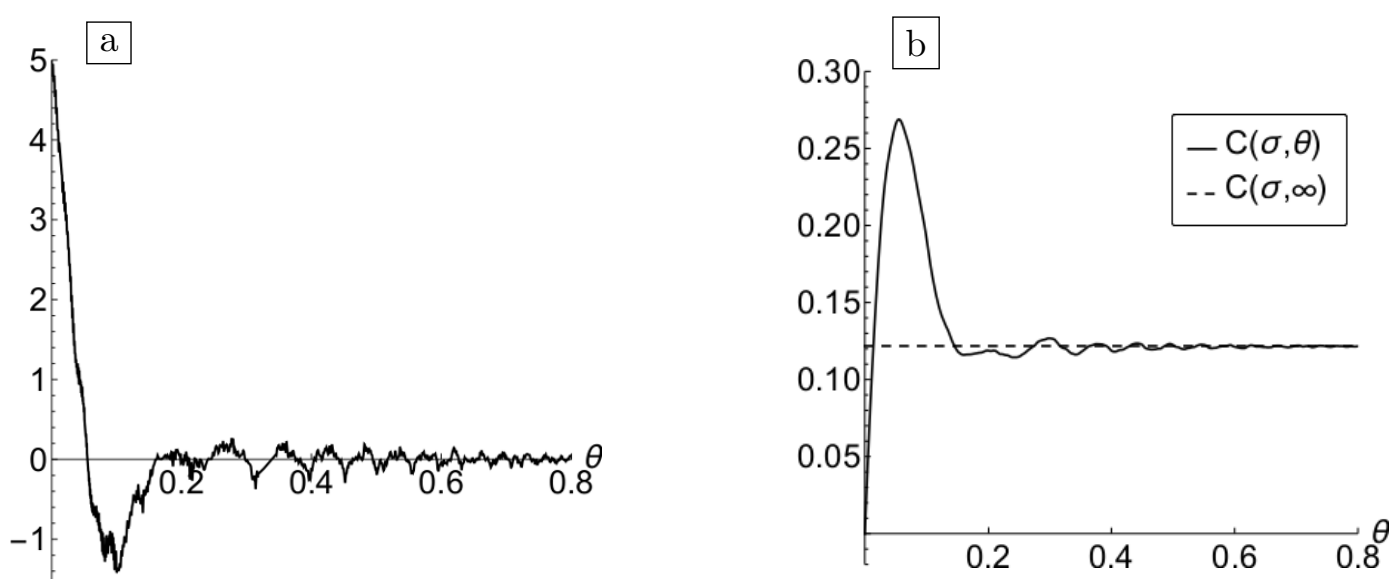

Figure 5. a) Example integrand to be integrated over to calculate $\partial \mathcal{I}_{-} / \partial \sigma$. b) The corresponding cumulative distribution function.

probability, which is defined for asymptotic states, is positive. Therefore in general, $\partial \mathrm{P} / \partial \sigma$ cannot be interpreted as a rate. In figure 4 , we note that the introduction of strong field gradients through a sharper magnetic field edge, allows for an increase in the amplitude of $\partial \mathrm{P} / \partial \sigma$ near this edge. One way this can be understood is by considering the Fourier transform of the limit $\Phi \rightarrow 0$, which would give a frequency spectrum $\sim \operatorname{sinc}\left(r \varkappa^{0} L\right)$, where $r$ is a real number. The opposite limit $\Phi \rightarrow \infty$ makes the field formally constant resulting in a delta frequency spectrum at the origin. Thus, the higher the field gradient, the larger the contribution from higher frequencies, which can bridge the gap to the $2 \mathrm{~m}$ pair-creation threshold, thereby reducing the necessary tunneling time and increasing the probability. This is similar to the situation of pair-creation by a photon in the background of a plane-wave laser pulse, where shorter pulses were found to drastically increase paircreation probabilities $[20,21]$. Although homogeneous magnetic field strengths are limited to around $O(1-10) \mathrm{T}$ in the lab, there is increasing interest in the quasi-static fields of the order of $\sim 10^{5} \mathrm{~T}$ that are generated in intense laser-plasma collisions [72].

To demonstrate the numerical integration in $\theta$, we plot in figure $5 \mathrm{a}$ how the integrand in eq. (4.2) depends on $\theta$, with the same parameters as the central plot in figure $4\left(\delta^{2}=0\right.$, $\Phi=0.05, \xi=10, \eta_{k}=0.1$ ) evaluated at $\sigma=0.3$ (as the problem is symmetric around $\theta=0$, we have simply calculated points in the region $\theta \geq 0$ ). The convergence of the integral is indicated in figure $5 \mathrm{~b}$ where the cumulative distribution function $C(\sigma, \theta)$, given by

$$
C(\sigma, \theta)=\int_{0}^{\theta} \frac{\partial^{2} \mathcal{I}_{-}(\sigma, y)}{\partial \sigma \partial y} d y
$$

is plotted and tends towards a constant as the upper integration bound in $\theta$ is increased.

Figure 5 shows the major contribution to the $\theta$-integral originates from $\theta \ll 1$ and how the frequency of oscillation increases with $\theta$. It is instructive to compare figure 5 with a point outside the sharp edges (at $\sigma= \pm 0.5$ ) of the magnetic field, to illustrate differences between the full result and the LCFA. Consider the sharper magnetic field in the right-hand plot of figure 4 (where $\delta^{2}=0, \Phi=0.01, \xi=10, \eta_{k}=0.1$ ) for $\sigma=0.575$. According to the 

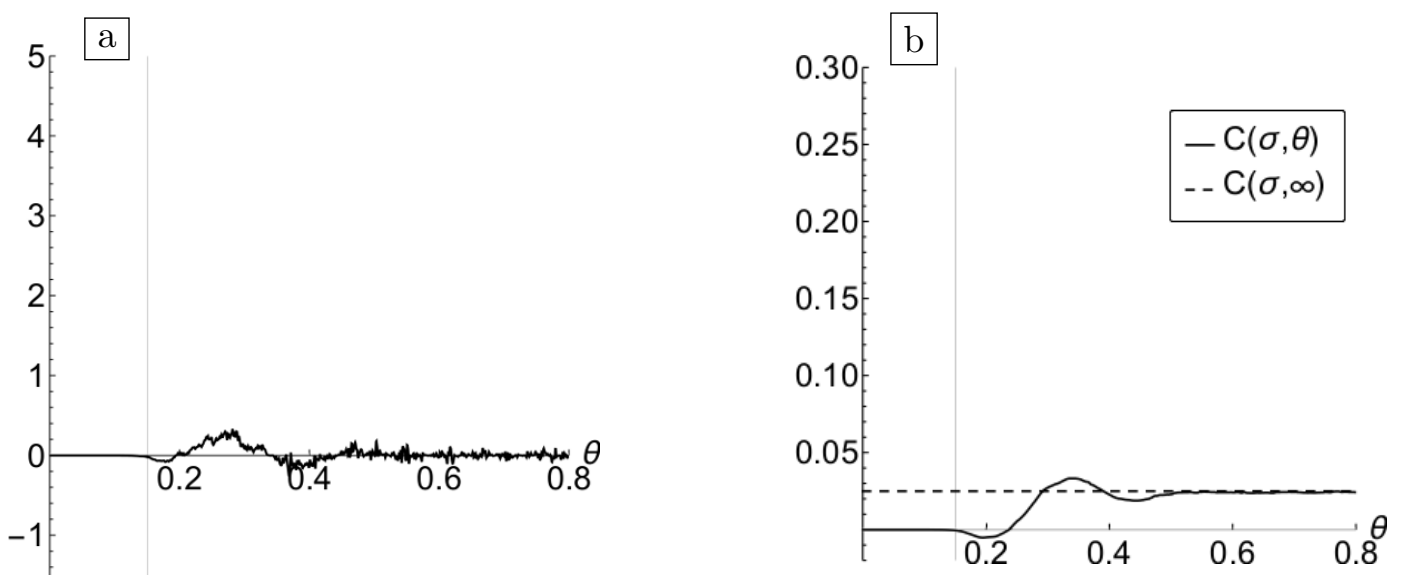

Figure 6. a) A plot of $\partial^{2} \mathcal{I}_{-} / \partial \sigma \partial \theta$ at $\sigma=0.575$ for the magnetic field with $\xi=10, \eta_{k}=0.1$ and $\Phi=0.01$. b) The corresponding cumulative distribution function. The vertical line in the plots is at $\theta / 2=\sigma-0.5$, i.e. the distance away from the edge of the magnetic field.

LCFA, there is no contribution here, but as is clear from figure 4, the full probability does actually contribute. Plotting $\partial P / \partial \sigma$ in figure 6 , we see that indeed in the full probability there is zero contribution around $\theta=0$, however, at precisely the value that $\sigma-\theta / 2<0.5$, the integral begins to contribute with increasing $\theta$ (recall that the Kibble mass in eq. (2.14) is defined using a window average eq. (2.10) evaluated for phases between $\sigma \pm \theta / 2$ ).

The LCFA is an expansion in small $\theta$ and therefore at $\sigma=0.575$ it is not sensitive to contributions from the field at $\sigma=0.5$, even if the field changes a substantial amount at this point.

Finally in figure 7 , we give some examples of the LCFA probability for pair-creation in a magnetic field in the above-threshold case. We choose $\Phi=0.2$, as this was where the numerics and LCFA agreed well for the massless case.

In figure $7 \mathrm{a}$ we note that, as the axion mass is increased, the effect of the magnetic field is one from increasing axion decay $\left(\delta^{2} \leq 4\right)$, to the point where the magnetic field only slightly modifies the probability, and in fact in some regions of parameter space suppresses it. In figure $7 \mathrm{~b}$, we see that as the energy parameter is increased for a typical above-thethreshold scenario $\left(\delta^{2}=4.1\right)$, the effect of the magnetic field enhances pair decay. As the axion impinges from the vacuum in the magnetic field, the transition in the probability from vacuum decay to field-assisted decay is nontrivial, and depending on the axion's energy, can also lead to a suppression of the decay probability.

\section{Example experimental scenarios}

The results of the previous section can be used to provide an estimate for exclusion bounds that could be measured at typical upcoming experiments. One example is the LUXE experiment at DESY [73], which will combine the $17.5 \mathrm{GeV}$ electron beam that drives the European XFEL laser, with a thin-foil target to produce Bremsstrahlung, which can be used as a probe in photon-laser collisions [74]. The set-up we envisage is a variation of 

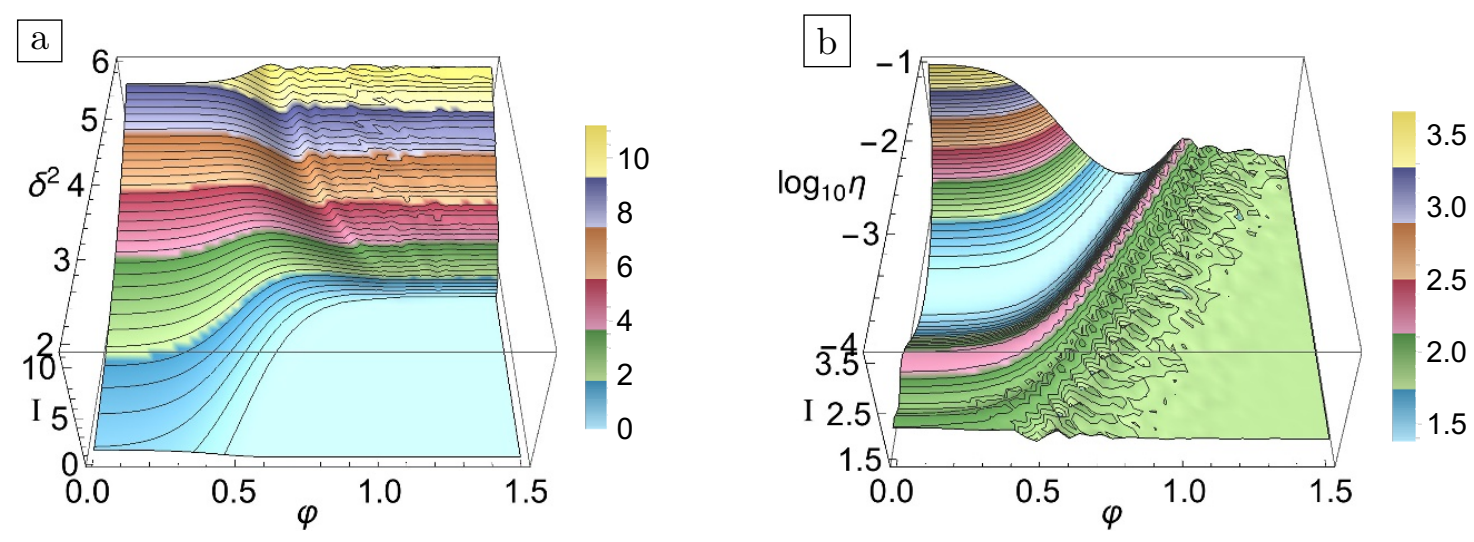

Figure 7. Plots for $\mathcal{I}_{-}^{\text {LCFA }}$ edges for the first case in figure 4 , with $\eta_{k}=0.1, \xi=10, \Phi=0.2$, $\delta^{2}=4.1$ (unless these parameters are varied in the plot). Left: the transition from below- to above-the-threshold behaviour. Right: the role of the energy parameter in increasing decay in the magnetic field region. (The colours represent the vertical axis height as denoted by the scale alongside each plot.)

a typical LSW experiment, where the generation mechanism is axionic bremsstrahlung in the thin-foil target, and regeneration of the axions occurs behind some thick shielding, a distance away.

In the first scenario, we consider the regeneration to occur in a magnetic field. If we take as parameters for the magnet, those using in the ALPs experiment at DESY [10], of $B=5 \mathrm{~T}$ and $L=4.21 \mathrm{~m}$ then using eq. (4.3), as long as the field decays over a distance greater than $2 \mathrm{~mm}$, which we will assume, the LCFA can be used.

We assume the collision is head-on with the magnetic field wavevector. To estimate the regeneration rate of axions, we take the thin-target approximation to bremsstrahlung from [74], and multiply by $g^{2} / e^{2}$ to acquire an approximate rate of axionic bremsstrahlung:

$$
k^{-} \frac{d N\left(k^{-}\right)}{d k^{-}} \approx \frac{g^{2}}{e^{2}} \frac{X}{X_{0}}\left[\frac{4}{3}-\frac{4}{3} \frac{k^{-}}{p^{-}}+\left(\frac{k^{-}}{p^{-}}\right)^{2}\right] .
$$

The number of axions regenerated is then:

$N_{\phi}=\left.N_{e} N_{\text {shots }}\left(\frac{g^{2}}{4 \pi}\right)^{2} \frac{X}{X_{0}} \frac{1}{2 \gamma_{e}} \frac{L}{\bar{\lambda}} \int_{0}^{1} \frac{d x}{x^{2}}\left(\frac{4}{3}-\frac{4}{3} x+x^{2}\right) \int_{0}^{1} d t \frac{\partial \mathrm{R}_{-}^{\mathrm{CCF}}\left(\chi_{k}, \delta^{2}, t\right)}{\partial t}\right|_{\chi_{k}=2 \gamma_{e} \frac{B}{B_{Q}} x}$

where $\gamma_{e} \approx 3.5 \times 10^{4}$ is the relativistic gamma factor of the electrons hitting the foil, an $X / X_{0}$ is the average number of radiation lengths undergone in the foil (we take $X / X_{0}=$ 0.1 ). For the number of shots, we assume 24 hours of continuous operation and a frequency of $1 \mathrm{~Hz}$ for collisions, and take the number of electrons to be $N_{e}=10^{9}$. For values of $\delta \geq 2$, the rate of regeneration is almost identical to that without a magnetic field. For $\delta<2$, there is a small region of parameter space where the magnetic field field can cause regeneration to 

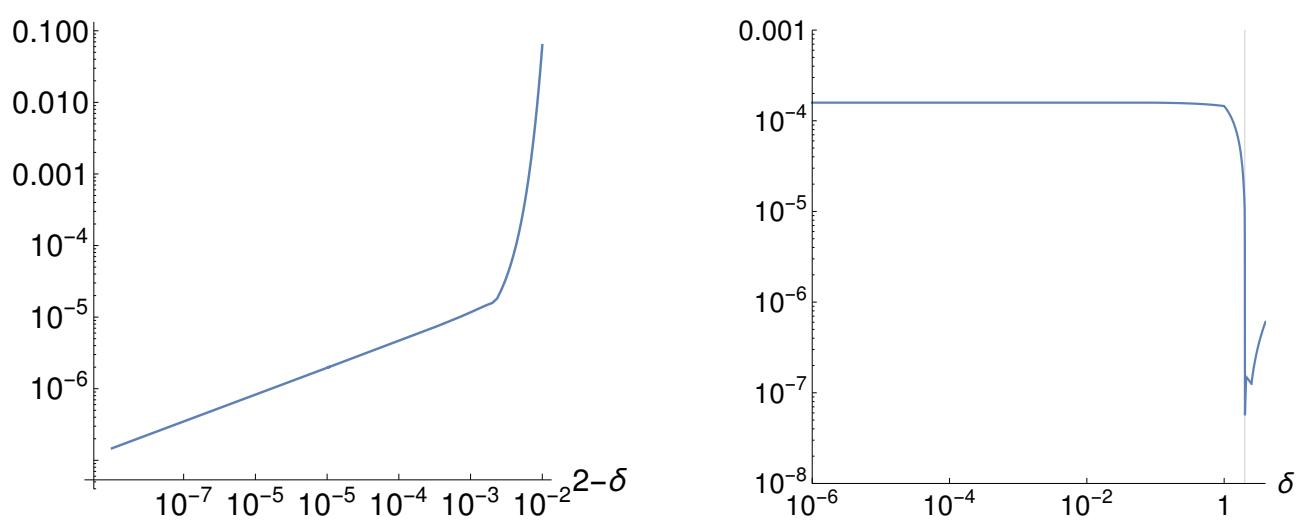

Figure 8. Projected exclusion bounds for $g_{\phi e}$ in the magnetic set-up (left) and laser set-up (right).

occur where it is forbidden by vacuum (see the left-hand plot of figure 8 ). So with this setup, although the detection region is always "on", pair-creation is exponentially suppressed unless the axion is above-threshold. This set-up is more suitable for axion regeneration via the di-photon coupling.

In the second scenario, we take the regeneration to occur in an intense laser pulse, which is synchronised with the firing of the electron beam such that the pulse of generated axions arrives at the centre of the laser pulse. Assuming a plane-wave laser pulse of intensity parameter $\xi=10$ and pulse duration $\Phi=50$, using a similar analysis, we find the exclusion bounds in the right-hand plot of figure 8 .

\section{Summary}

The decay of a massive ALP into an electron-positron pair in a high intensity EM background field has been investigated. An example derivation for the case of massive axions was presented, and the results for massive scalars and vector bosons were also given. Although the pre-exponents of scalars and vectors are different to the pseudoscalar case, the nonpertubative exponential dependency and kinematics are identical and so we expect our results to have significance for these cases as well. A constant crossed field was chosen as an example background to investigate how the decay depends on experimental and axion parameters. Three distinct regions were identified: i) below threshold decay, where the rate of decay was via tunneling through the background field; ii) above threshold decay which can proceed in the absence of a background field; iii) strong-field limit, where the concept of a threshold disappears. With interest in lab measurement in a constant magnetic field, we calculated the decay probability in a quasi-constant crossed field of finite spatial extent. This is expected to be a good approximation to decay in a constant magnetic field for highly-relativistic seed ALP particles. Using a phenomenological model, the effect of field "edges" and hence strong field gradients was investigated.

In below-threshold decay, a new mass-dependent tunneling exponent was identified, which shows how the gap to the threshold pair-creation energy of $2 m$ is partially bridged by the mass of the axion. This is reminiscent of Schwinger pair production catalysis [75] 
by a second, higher-frequency background overlaid on a constant background, where here, the ALP mass plays the role of the higher-frequency background. The same expression for the tunnelling exponent was arrived at independently by using simple energy-momentum arguments in $[69,70]$.

The case of above-threshold decay shows an interplay between the two channels of: i) vacuum decay and ii) field-stimulated decay through a nonperturbative dependence on $\chi_{k}$ (a combination of external-field and ALP particle parameters). The field was found to both increase and decrease the probability for ALP decay, due to it inducing an oscillation in the probability around the vacuum value. This oscillation is in the ALP mass parameter, but also in $\chi_{k}$ and hence the field strength and ALP lightfront momentum. An asymptotic formula for the oscillations was found using a stationary phase analysis, and so, even if the background is not a constant crossed field, it is expected that such oscillations are a general characteristic of the above-threshold decay of ALPs into electron-positron pairs in plane-wave fields.

In the strong-field regime, $\chi_{k} \gg 1$, which would be challenging to arrive at in the lab, but may have significance in some astrophysical scenarios, the concept of a threshold disappears, and the nonperturbative asymptotic result depends only on the field. This is to be expected - eventually if the field is strong enough, the vacuum decay channel is negligibly small, and so loses meaning.

To investigate a quasi-constant crossed field of finite spatial extent in the lab, we introduced a field with dimensionless "sharpness" parameter, $\Phi$, parametrising the departure from a top-hat shape at $\Phi=0$. We found that the sharper the field, the worse the approximation of taking the probability rate for a constant field and integrating it over the field shape (the so-called locally-constant field approximation). In particular, there are still contributions from outside of the field, which we found to be traceable to interference over the trajectory of the seed particle, which are absent from the simple approximation. Finally, identifying a region where the locally constant field approximation was valid, we presented the nontrivial dependency of the decay probability in a magnetic field with edge, as the mass of the decaying particle crosses the vacuum decay threshold.

\section{Acknowledgments}

The authors thank Subir Sarkar for suggested improvements to the manuscript. B. K. acknowledges computational resources supplied by A. Ilderton. B. K. and B. M. D. acknowledge funding from Grant No. EP/P005217/1. K. A. B. and G. G. would like to thank AWE plc for support.

Open Access. This article is distributed under the terms of the Creative Commons Attribution License (CC-BY 4.0), which permits any use, distribution and reproduction in any medium, provided the original author(s) and source are credited. 


\section{References}

[1] R.D. Peccei and H.R. Quinn, CP Conservation in the Presence of Instantons, Phys. Rev. Lett. 38 (1977) 1440 [INSPIRE].

[2] A. De Angelis, M. Roncadelli and O. Mansutti, Evidence for a new light spin-zero boson from cosmological gamma-ray propagation?, Phys. Rev. D 76 (2007) 121301 [arXiv:0707.4312] [INSPIRE].

[3] A. Mirizzi, G.G. Raffelt and P.D. Serpico, Photon-Axion Conversion in Intergalactic Magnetic Fields and Cosmological Consequences, Springer, Heidelberg Germany (2008), pg. 115 .

[4] M. Simet, D. Hooper and P.D. Serpico, The Milky Way as a Kiloparsec-Scale Axionscope, Phys. Rev. D 77 (2008) 063001 [arXiv:0712.2825] [INSPIRE].

[5] M.A. Sanchez-Conde, D. Paneque, E. Bloom, F. Prada and A. Domínguez, Hints of the existence of Axion-Like-Particles from the gamma-ray spectra of cosmological sources, Phys. Rev. D 79 (2009) 123511 [arXiv:0905.3270] [INSPIRE].

[6] E. Bulbul, M. Markevitch, A. Foster, R.K. Smith, M. Loewenstein and S.W. Randall, Detection of An Unidentified Emission Line in the Stacked X-ray spectrum of Galaxy Clusters, Astrophys. J. 789 (2014) 13 [arXiv:1402.2301] [INSPIRE].

[7] A. Boyarsky, O. Ruchayskiy, D. Iakubovskyi and J. Franse, Unidentified Line in X-Ray Spectra of the Andromeda Galaxy and Perseus Galaxy Cluster, Phys. Rev. Lett. 113 (2014) 251301 [arXiv: 1402.4119] [INSPIRE].

[8] J. Jaeckel, J. Redondo and A. Ringwald, $3.55 \mathrm{keV}$ hint for decaying axionlike particle dark matter, Phys. Rev. D 89 (2014) 103511 [arXiv:1402.7335] [INSPIRE].

[9] J. Redondo and A. Ringwald, Light shining through walls, Contemp. Phys. 52 (2011) 211 [arXiv: 1011.3741] [INSPIRE].

[10] K. Ehret et al., New ALPS Results on Hidden-Sector Lightweights, Phys. Lett. B 689 (2010) 149 [arXiv: 1004.1313] [INSPIRE].

[11] R. Bähre et al., Any light particle search II - Technical Design Report, 2013 JINST 8 T09001 [arXiv: 1302.5647] [INSPIRE].

[12] L. Capparelli, G. Cavoto, J. Ferretti, F. Giazotto, A.D. Polosa and P. Spagnolo, Axion-like particle searches with sub-THz photons, Phys. Dark Univ. 12 (2016) 37 [arXiv:1510.06892] [INSPIRE].

[13] CAST collaboration, New CAST limit on the axion-photon interaction, Nature Phys. 13 (2017) 584 .

[14] E. Armengaud et al., Conceptual Design of the International Axion Observatory (IAXO), 2014 JINST 9 T05002 [arXiv:1401.3233] [INSPIRE].

[15] A.V. Borisov and V.Y. Grishina, Compton production of axions on electrons in a constant external field, Sov. Phys. JETP 83 (1996) 868.

[16] K. Barth et al., CAST constraints on the axion-electron coupling, JCAP 05 (2013) 010 [arXiv: 1302.6283] [INSPIRE].

[17] J.S. Toll, The dispersion relation for light and its application to problems involving electron pairs, Ph.D. Thesis, Princeton University, Princeton U.S.A. (1952). 
[18] V.N. Baier and V.M. Katkov, Pair creation by a photon in a strong magnetic field, Phys. Rev. D 75 (2007) 073009 [hep-ph/0701119] [INSPIRE].

[19] T. Heinzl, A. Ilderton and M. Marklund, Finite size effects in stimulated laser pair production, Phys. Lett. B 692 (2010) 250 [arXiv:1002.4018] [INSPIRE].

[20] A.I. Titov, B. Kämpfer, H. Takabe and A. Hosaka, Breit-Wheeler process in very short electromagnetic pulses, Phys. Rev. A 87 (2013) 042106 [arXiv:1303.6487] [INSPIRE].

[21] T. Nousch, D. Seipt, B. Kämpfer and A.I. Titov, Pair production in short laser pulses near threshold, Phys. Lett. B 715 (2012) 246 [INSPIRE].

[22] V.I. Ritus, Effect of an electromagnetic field on decays of elementary particles, Zh. Eksp. Teor. Fiz. 56 (1969) 986.

[23] W. Becker et al., A note on total cross sections and decay rates in the presence of a laser field, Phys. Lett. A 94 (1983) 131.

[24] N.B. Narozhny and A.M. Fedotov, Comment on 'Laser-Assisted Muon Decay', Phys. Rev. Lett. 100 (2008) 219101 [INSPIRE].

[25] F.V. Bunkin and I.I. Tugov, Possibility of Creating Electron-Positron Pairs in a Vacuum by the Focusing of Laser Radiation, Sov. Phys. Dokl. 14 (1970) 678.

[26] V.S. Popov, Pair production in a variable and homogeneous electric field as an oscillator problem, Sov. Phys. JETP 35 (1972) 659.

[27] G.V. Dunne, Heisenberg-Euler effective Lagrangians: Basics and extensions, in From fields to strings: Circumnavigating theoretical physics. Ian Kogan memorial collection (3 volume set), M. Shifman, A. Vainshtein and J. Wheater eds., World Scientific, New York U.S.A. (2004), pg. 445 [hep-th/0406216] [INSPIRE].

[28] K. Sogut, H. Yanar and A. Havare, Production of Dirac Particles in External Electromagnetic Fields, Acta Phys. Polon. B 48 (2017) 1493 [arXiv:1703. 07776] [InSPIRE].

[29] G. Piccinelli and A. Sanchez, Magnetic Field Effect on Charged Scalar Pair Creation at Finite Temperature, Phys. Rev. D 96 (2017) 076014 [arXiv:1707.08257] [INSPIRE].

[30] M. Coppola, D. Gomez Dumm, S. Noguera and N.N. Scoccola, Neutral and charged pion properties under strong magnetic fields in the NJLS model, Phys. Rev. D 100 (2019) 054014 [arXiv: 1907.05840] [INSPIRE].

[31] V.I. Ritus, Quantum effects of the interaction of elementary particles with an intense electromagnetic field, J. Russ. Laser Res. 6 (1985) 497.

[32] Yu.I. Klimenko, O.S. Pavlova and E.Yu. Klimenko, $\tau$-Lepton decay in an arbitrary plane-wave electromagnetic field, Sov. Phys. J. 28 (1985) 972.

[33] A.V. Kurilin, Leptonic decays of the $W$ boson in a strong electromagnetic field, Phys. Atom. Nucl. 67 (2004) 2095 [arXiv: 0709.0335] [INSPIRE].

[34] A.V. Kurilin, Z0-boson decays in a strong electromagnetic field, Phys. Atom. Nucl. 72 (2009) 1034 [arXiv: 1309.2780] [INSPIRE].

[35] S. Villalba-Chávez and C. Muller, Photo-production of scalar particles in the field of a circularly polarized laser beam, Phys. Lett. B 718 (2013) 992 [arXiv:1208.3595] [INSPIRE].

[36] D.A. Burton and A. Noble, Plasma-based wakefield accelerators as sources of axion-like particles, New J. Phys. 20 (2018) 033022 [arXiv:1710.01906] [InSPIRE]. 
[37] B. King, Electron-seeded ALP production and ALP decay in an oscillating electromagnetic field, Phys. Lett. B 782 (2018) 737 [arXiv:1802.07507] [InSPIRE].

[38] B.M. Dillon and B. King, ALP production through non-linear Compton scattering in intense fields, Eur. Phys. J. C 78 (2018) 775 [arXiv:1802.07498] [INSPIRE].

[39] B.M. Dillon and B. King, Light scalars: coherent nonlinear Thomson scattering and detection, Phys. Rev. D 99 (2019) 035048 [arXiv:1809.01356] [INSPIRE].

[40] A.K. Harding and D. Lai, Physics of Strongly Magnetized Neutron Stars, Rept. Prog. Phys. 69 (2006) 2631 [astro-ph/0606674] [INSPIRE].

[41] W.H. Furry, On Bound States and Scattering in Positron Theory, Phys. Rev. 81 (1951) 115 [INSPIRE].

[42] D.M. Wolkow, Uber eine Klasse von Losungen der Diracschen Gleichung, Z. Phys. 94 (1935) 250 [INSPIRE].

[43] M. Marklund and P.K. Shukla, Nonlinear collective effects in photon-photon and photon-plasma interactions, Rev. Mod. Phys. 78 (2006) 591 [hep-ph/0602123] [INSPIRE].

[44] H. Gies, Strong laser fields as a probe for fundamental physics, Eur. Phys. J. D 55 (2009) 311 [arXiv:0812.0668] [inSPIRE].

[45] A. Di Piazza and A.I. Milstein, Quasiclassical approach to high-energy QED processes in strong laser and atomic fields, Phys. Lett. B 717 (2012) 224 [arXiv:1204.2502] [INSPIRE].

[46] N.B. Narozhny and A.M. Fedotov, Extreme light physics, Contemp. Phys. 56 (2015) 249 [INSPIRE].

[47] D.L. Burke et al., Positron production in multi-photon light by light scattering, Phys. Rev. Lett. 79 (1997) 1626 [INSPIRE].

[48] C. Bamber et al., Studies of nonlinear QED in collisions of 46.6-GeV electrons with intense laser pulses, Phys. Rev. D 60 (1999) 092004 [INSPIRE].

[49] B. Döbrich and H. Gies, Axion-like-particle search with high-intensity lasers, JHEP 10 (2010) 022 [arXiv: 1006.5579] [INSPIRE].

[50] S. Villalba-Chávez, Laser-driven search of axion-like particles including vacuum polarization effects, Nucl. Phys. B 881 (2014) 391 [arXiv:1308.4033] [INSPIRE].

[51] S. Villalba-Chávez, T. Podszus and C. Müller, Polarization-operator approach to optical signatures of axion-like particles in strong laser pulses, Phys. Lett. B 769 (2017) 233 [arXiv: 1612.07952] [INSPIRE].

[52] J.T. Mendonca, Axion excitation by intense laser fields, EPL 79 (2007) 21001 [hep-ph/0702091] [INSPIRE].

[53] C.N. Harvey, A. Ilderton and B. King, Testing numerical implementations of strong field electrodynamics, Phys. Rev. A 91 (2015) 013822 [arXiv:1409.6187] [INSPIRE].

[54] A. Di Piazza, M. Tamburini, S. Meuren and C.H. Keitel, Implementing nonlinear Compton scattering beyond the local constant field approximation, Phys. Rev. A 98 (2018) 012134 [arXiv: 1708.08276] [INSPIRE].

[55] A. Ilderton, B. King and D. Seipt, Extended locally constant field approximation for nonlinear Compton scattering, Phys. Rev. A 99 (2019) 042121 [arXiv:1808.10339] [INSPIRE]. 
[56] B. King, A uniform locally constant field approximation for photon-seeded pair production, arXiv: 1908.06985 [INSPIRE].

[57] V. Dinu, T. Heinzl, A. Ilderton, M. Marklund and G. Torgrimsson, Vacuum refractive indices and helicity flip in strong-field QED, Phys. Rev. D 89 (2014) 125003 [arXiv:1312.6419] [INSPIRE].

[58] F.W.J. Olver, Asymptotics and Special Functions (AKP Classics), AK Peters, Natick U.S.A. (1997).

[59] A.I. Nikishov and V.I. Ritus, Quantum Processes in the Field of a Plane Electromagnetic Wave and in a Constant Field 1, Sov. Phys. JETP 19 (1964) 529 [inSPIRE].

[60] T. Heinzl and A. Ilderton, A Lorentz and gauge invariant measure of laser intensity, Opt. Commun. 282 (2009) 1879.

[61] A. Di Piazza, M. Tamburini, S. Meuren and C.H. Keitel, Implementing nonlinear Compton scattering beyond the local constant field approximation, Phys. Rev. A 98 (2018) 012134 [arXiv: 1708.08276] [INSPIRE].

[62] N.V. Elkina et al., QED cascades induced by circularly polarized laser fields, Phys. Rev. ST Accel. Beams 14 (2011) 054401 [arXiv: 1010.4528] [INSPIRE].

[63] B. King and H. Ruhl, Trident pair production in a constant crossed field, Phys. Rev. D 88 (2013) 013005 [arXiv: 1303.1356] [INSPIRE].

[64] J.D. Jackson, Classical Electrodynamics, thirrd edition, John Wiley \& Sons, Inc., New York U.S.A. (1999).

[65] C.F. von Weizsacker, Radiation emitted in collisions of very fast electrons, Z. Phys. 88 (1934) 612 [INSPIRE].

[66] E.J. Williams, Nature of the high-energy particles of penetrating radiation and status of ionization and radiation formulae, Phys. Rev. 45 (1934) 729 [INSPIRE].

[67] T. Heinzl, A. Ilderton and B. King, Classical and quantum particle dynamics in univariate background fields, Phys. Rev. D 94 (2016) 065039 [arXiv: 1607.07449] [INSPIRE].

[68] H.R. Reiss, Absorption of Light by Light, J. Math. Phys. 3 (1962) 59.

[69] E.K. Akhmedov, Beta decay and other processes in strong electromagnetic fields, Phys. Atom. Nucl. 74 (2011) 1299 [arXiv:1011.3776] [INSPIRE].

[70] A.M. Fedotov, Qualitative considerations in Intense Field QED, arXiv:1507.08512 [INSPIRE].

[71] C.M. Bender and S.A. Orszag, Advanced Mathematical Methods for Scientists and Engineers, Springer, Heidelberg Germany (1978).

[72] O. Jansen et al., Leveraging extreme laser-driven magnetic fields for gamma-ray generation and pair production, Plasma Phys. Control. Fusion 60 (2018) 054006.

[73] M. Altarelli et al., Summary of strong-field QED Workshop, arXiv:1905.00059 [INSPIRE].

[74] A. Hartin, A. Ringwald and N. Tapia, Measuring the Boiling Point of the Vacuum of Quantum Electrodynamics, Phys. Rev. D 99 (2019) 036008 [arXiv:1807.10670] [inSPIRE].

[75] G.V. Dunne, H. Gies and R. Schützhold, Catalysis of Schwinger Vacuum Pair Production, Phys. Rev. D 80 (2009) 111301 [arXiv:0908.0948] [InSPIRE]. 